\title{
Hommage à Francis Roussel
}

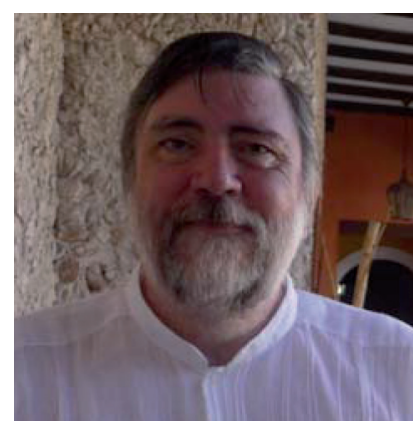

Francis nous a quitté brutalement le 6 avril 2014.

Nos pensées vont à son épouse Claudine, à ses enfants et ses petits-enfants.

Humaniste, déterminé, convaincu, travailleur, engagé socialement, visionnaire, disponible, bienveillant, homme érudit, voici quelques mots qui viennent immédiatement à l'esprit pour décrire Francis.

Francis a été étudiant en médecine à la faculté de Rouen. Il a obtenu son diplôme de docteur en médecine en 1975, puis son certificat d'études spécialisées d'hématologie en 1976 et son certificat d'études spécialisées d'anatomo-pathologie en juin 1978. Il sera nommé maître de conférences des universités et praticien hospitalier en 1988. Toute sa vie durant, il a exprimé sa fidélité à Rouen, sa ville.

Sa passion professionnelle était l'histologie. Il aimait l'enseigner. Expert reconnu en cytopathologie, notamment pulmonaire, il était un grand histologiste. Sa compétence était largement reconnue au sein de sa spécialité. Il a été membre de la sous section 4202 du Conseil national des universités pendant 12 ans, période pendant laquelle il s'est fait remarquer par la justesse et l'humanité des analyses des candidatures qui lui étaient soumises.

Depuis de très nombreuses années, Francis était fortement engagé dans la collectivité rouennaise. Il a occupé de nombreuses fonctions collectives, hospitalières et facultaires. Il fut membre de la Commission médicale d'établissement, du bureau de celle-ci et du Conseil d'administration du Centre hospitalier universitaire de Rouen. Pendant plus de 10 ans, Francis a aussi été membre du Conseil d'administration du Syndicat national des hospitalo-universitaires (SNHU). Pendant plusieurs années, il a été membre du Conseil scientifique de l'université pour y représenter et souvent y défendre les intérêts de la faculté de médecine. Récemment, il a été membre du Conseil de gestion de la Faculté de médecine de Rouen de 2008 à 2013, sous la présidence du Doyen Fréger. Francis a été un des acteurs clés de la réforme pédagogique à Rouen, à la fin des années 90, qui a permis à la Faculté de médecine de Rouen de prendre le tournant d'une pédagogie moderne, centrée sur l'étudiant. Acteur passionné des activités d'apprentissage par problèmes à Rouen, il a su accompagner leur mise en place il y a 15 ans.

Francis portait, chevillé au corps, le besoin constant d'innover et de développer de nouvelles approches et de nouveaux outils en éducation médicale. Au sein du Conseil pédagogique de la faculté, dont il était membre depuis 15 ans, et de la Commission «Évaluation et recherche en pédagogie », il a joué un rôle majeur. Il a ainsi participé à la préparation, la mise en ouvre et au suivi de nombreuses actions telles que l'évaluation des dispositifs pédagogiques d'apprentissage par problèmes, la mise au point de recommandations pour le fonctionnement d'un jury, l'évaluation des stages hospitaliers puis celles des enseignements. Son dynamisme était inépuisable. Ainsi, lors de la dernière réunion de la commission en mars dernier, consacrée à la préparation de l'évaluation de la formation du troisième cycle des études médicales, Francis, toujours en avance sur le groupe, a su nous convaincre d'aller au delà de 
l'évaluation des stages et de nous intéresser réellement à la qualité de la formation des internes durant leur formation à Rouen. Dès le lendemain de la commission, il rédigeait une proposition très pertinente, dans ce sens.

Francis a été un pédagogue dynamique. Au delà de ses grandes qualités d'enseignant, il a toujours su établir un dialogue et une proximité avec les étudiants et a œuvré pour les impliquer activement dans le processus de préparation des innovations pédagogiques. Nos étudiants lui en sont reconnaissants, leurs représentants se sont manifestés, à l'annonce de son décès, pour exprimer, auprès des instances facultaires, leur émotion et leur tristesse.

Le caractère novateur de ses travaux de recherche en pédagogie est indéniable. Il a été un des premiers à tester et évaluer le test de concordance de script en médecine générale, puis à proposer un test de concordance de script automatisé en ligne. Il a travaillé sur l'utilisation et l'intérêt des vidéos-questions à choix multiple comme outil d'évaluation dans les premières années du cursus médical. Il a été aussi un des premiers à évaluer la difficulté de correction du résumé lors d'une épreuve de lecture critique d'article ou encore à tester la faisabilité d'une épreuve de lecture critique d'article en ligne, préfigurant les épreuves classantes nationales informatisées (iECN) avant l'heure.

Depuis la mise en place des épreuves classantes nationales, Francis a travaillé sur les classements des étudiants rouennais, en comparaison avec les classements nationaux. Toujours performant dans ses analyses, il a su attirer l'attention sur le profil des étudiants qui réussissaient aux épreuves classantes nationales ou encore sur la corrélation entre le nombre d'enseignants par faculté et les résultats aux épreuves classantes nationales. Son analyse scientifique et critique était toujours pertinente, avisée et teintée d'humour. Lors d'un séminaire du diplôme inter-universitaire de pédagogie médicale à Rouen, sa présentation s'intitulait : "Les ECN : un OPNI » (pour Objet Pédagogique Non Identifié).
Bien sûr, toute cette dynamique de recherche s'est traduite par une intense activité de publications scientifiques en éducation médicale, associée à la présentation de communications dans des congrès internationaux. Beaucoup d'entre nous ont rencontré, échangé et sympathisé avec Francis lors des conférences francophones de pédagogie médicale. Il a présenté de nombreuses communications lors de conférences de la Conférence internationale des doyens et des facultés de médecine d'expression française (CIDMEF), de la Société internationale francophone d'éducation médicale (SIFEM) ou encore des Forums de pédagogie médicale au Québec. Au Congrès de la SIFEM à Beyrouth en 2006, ses travaux en pédagogie médicale lui ont valu de recevoir le prix du Conseil scientifique pour sa communication : "Pour une transformation des épreuves classantes nationales en un examen classant national », communication qui a fait l'objet d'une publication la même année dans Pédagogie Médicale.

C'est une figure emblématique de la vie hospitalière et universitaire de Rouen et un grand acteur du monde de la pédagogie médicale que nous perdons. Homme d'idées et d'actions, nous mesurons combien nous perdons un collègue cher dont le talent de pédagogue et les visions humanistes ont marqué la vie de la communauté médicale et universitaire rouennaise.

Joël LADNER, Jean François GEHANNO, Christophe GIRAULT, Agnès LIARD, Olivier MOUTERDE, Louis SIBERT.

La Commission «Évaluation et recherche en pédagogie médicale »,

Conseil pédagogique, UFR de médecine et pharmacie de Rouen

Benoit VEBER,

Vice-doyen à la pédagogie, UFR de médecine et pharmacie de Rouen 